\title{
A Stable Quotient-Difference Algorithm
}

\author{
By A. N. Stokes
}

\begin{abstract}
This paper shows how the arithmetic of the quotient-difference algorithm can be performed using the forward and backward difference tables of each column. This removes the tendency of the algorithm to amplify errors. As an application, 70 continued fraction coefficients are calculated for the modified Bessel function $K_{0}(z)$ in single-precision arithmetic. There is no significant build-up of error.
\end{abstract}

Introduction. A quotient-difference algorithm is formed in the following way [2]. Two columns of numbers $e_{0}^{(n)}$ and $q_{1}^{(n)}$ are given. The table is continued using the relations

$$
e_{k}^{(n)}-e_{k-1}^{(n+1)}=q_{k}^{(n+1)}-q_{k}^{(n)}, \quad q_{k+1}^{(n)} e_{k}^{(n)}=e_{k}^{(n+1)} q_{k}^{(n+1)},
$$

possibly with some boundary relations. Stability problems arise because of the formation of differences in the $q$-columns. The instability cannot be removed by rearranging the procedure; it can be shown that elements of the later columns do depend on higherorder differences of the columns used earlier.

Some methods of overcoming instability are described in [1]. They are referred to in the example below; none are very successful.

The present approach overcomes the problem by doing the calculations with higher-order differences. Each column is stored as a set of differences, and the set of differences characterizing a new column is formed from earlier differences in such a way that accuracy is not lost.

It is necessary that the differences of the initial columns should be accurate, so they cannot be formed simply by subtraction. If the initial columns are given by an analytic relation, the higher-order differences can probably be derived from this too.

The stabilized algorithm is useful for calculating continued fraction coefficients. Its usefulness is extended by the demonstration in [3] that the q-d algorithm can be used to produce continued fractions approximating series expansions for a function about two different points. Such fractions can be used as global approximations in the region between the points.

Difference Arithmetic. Consider two sets of numbers $a=\left\{a_{i} ; i=0, \ldots, n\right\}$ and $b=\left\{b_{i} ; i=0, \ldots, n\right\}$. The following operations on such sets are defined: .

$$
\begin{aligned}
& a+b=\left\{a_{i}+b_{i} ; i=0, \ldots, n\right\}, \\
& a \otimes b=\left\{a_{i} b_{i} ; i=0, \ldots, n\right\},
\end{aligned}
$$

Received June 28, 1978; revised November 21, 1978.

AMS (MOS) subject classifications (1970). Primary 65D15; Secondary 41 A21. 


$$
\begin{aligned}
& \Delta(a)=\left\{a_{i+1}-a_{i} ; i=0, \ldots, n-1\right\}, \\
& L(a)=\left\{a_{i} ; i=1, \ldots, n\right\} .
\end{aligned}
$$

The symbol $\Delta^{k}(a)$ represents the result of $k$ applications of the operator $\Delta$, where $k \leqslant n$. Then $\Delta^{k}(a)=\left\{\Delta_{m}^{k}(a) ; m=0, \ldots, n-k\right\}$.

The array $\Delta_{m}^{k}(a)$ is called the difference table of the set $a$. Then the difference tables of the results of the above operations can be formed:

$$
\begin{aligned}
\Delta_{m}^{k}(a+b) & =\Delta_{m}^{k}(a)+\Delta_{m}^{k}(b), & & m=0, \ldots, n-k ; k=0, \ldots, n, \\
\Delta_{m}^{k}(L(a)) & =\Delta_{m+1}^{k}(a), & & m=0, \ldots, n-k-1 ; k=0, \ldots, n-1, \\
\Delta_{m}^{k}(\Delta a) & =\Delta_{m}^{k+1}(a), & m & =0, \ldots, n-k-1 ; k=0, \ldots, n-1,
\end{aligned}
$$

while for multiplication the following analogue of Leibniz's rule applies [4] :

$$
\Delta_{m}^{k}(a \otimes b)=\sum_{j=0}^{k}\left(\begin{array}{l}
k \\
j
\end{array}\right) \Delta_{m+k-j}^{j}(a) \Delta_{m}^{k-j}(b), \quad m=0, \ldots, n-k ; k=0, \ldots, n .
$$

The Algorithm. Suppose the columns $e_{0}^{(k)}, k=1, \ldots, N$, and $q_{1}^{(k)}, k=$ $0, \ldots, N$, together with their difference tables, are given to the required accuracy, where $N$ is even, for convenience. Suppose the relations mentioned previously are to be used without boundary conditions to form a q-d table, so

$$
e_{j}^{(k)}=e_{j-1}^{(k+1)}+q_{j}^{(k+1)}-q_{j}^{(k)}, \quad j=1, \ldots, N / 2, k=0, \ldots, N+1-2 j,
$$

and

$$
q_{j+1}^{(k)} e_{j}^{(k)}=q_{j}^{(k+1)} e_{j}^{(k+1)}, \quad j=1, \ldots, N / 2, k=0, \ldots, N-2 j .
$$

Let $e_{j}$ and $q_{j}$ represent, respectively, the sequences $\left\{e_{j}^{(k)} ; k=0,1, \ldots, N+1-2 j\right\}$ and $\left\{q_{j}^{(k)} ; k=0,1, \ldots, N-2 j+2\right\}$.

From (1), $e_{j}=L\left(e_{j-1}\right)+\Delta\left(q_{j}\right)$. So

$$
\begin{aligned}
\Delta_{n}^{m}\left(e_{j}\right)=\Delta_{n+1}^{m}\left(e_{j-1}\right) & +\Delta_{n}^{m+1}\left(q_{j}\right), \\
& m=0, \ldots, N+1-2 j, n=0, \ldots, N+1-2 j-m .
\end{aligned}
$$

From (2) $q_{j+1} \otimes e_{j}=L\left(q_{j}\right) \otimes L\left(e_{j}\right)$. So

$$
\Delta_{n}^{m}\left(q_{j+1} \otimes e_{j}\right)=\sum_{i=0}^{m}\left(\begin{array}{c}
m \\
j
\end{array}\right) \Delta_{m+n-j}^{i}\left(q_{j+1}\right) \Delta_{n}^{m-i}\left(e_{j}\right)
$$

$$
=\sum_{i=0}^{m}\left(\begin{array}{c}
m \\
i
\end{array}\right) \Delta_{m+n+1-j}^{i}\left(q_{j}\right) \Delta_{n+1}^{m-i}\left(e_{j}\right)
$$

for $m=0, \ldots, N-2 j ; n=0, \ldots, N-2 j-m$.

The algorithm uses Eqs. (3) and (4) in the following way. Suppose the difference tables for $e_{j-1}$ and $q_{j}$ are known. Then (3) gives the difference table for $e_{j}$, without forming differences of column elements by subtraction. Then Eq. (4) is solved for $\Delta_{N-2 j-m}^{m}\left(q_{j}\right)$ for $m=0,1, \ldots, N-2 j$. 
Then the full difference table for $q_{j+1}$ can be formed using the relations

$$
\Delta_{N-2 j-m}^{m-i}\left(q_{j+1}\right)=\Delta_{N-2 j-m}^{m-i+1}\left(q_{j+1}\right)+\Delta_{N-2 j-m-1}^{m-i}\left(q_{j+1}\right)
$$

for $m=i, \ldots, N-2 j ; i=1, \ldots, N-2 j$. This gives the values $q_{j+1}^{(k)}=\Delta_{k}^{0}\left(q_{j+1}\right)$, $k=0, \ldots, N-2 j$.

It is possible to avoid storing a full difference table at any time by retaining only the leading diagonals $\Delta_{N-2 j-m}^{m}\left(q_{j+1}\right)$ and $\Delta_{N-2 j+1-m}^{m}\left(e_{j}\right), m=0,1, \ldots$ It is necessary to form the required diagonals of the difference table of $e_{j}$ in (4), but this can be done sequentially, storing only one diagonal at a time.

The procedure uses more arithmetic than the usual way of implementing the q-d algorithm, but it eliminates the differencing of adjacent elements which makes the q-d algorithm unstable. There are approximately $N^{3} / 3$ additions and $N^{3} / 3$ multiplications of differences, and also $N^{3} / 3$ multiplications by binomial factors needed to form a full q-d table. The normal q-d algorithm uses $N^{2} / 2$ additions and $N^{2} / 2$ multiplications.

Application to Continued Fractions. To find the first $n$ coefficients $a_{i}$ of the continued fraction

$$
\frac{1}{\mid 1}+\frac{a_{1} x \mid}{\mid 1}+\frac{a_{2} x \mid}{\mid 1}+\cdots
$$

which corresponds to the power series $P=1+b_{1} x+b_{2} x^{2}+\cdots$ two initial columns of a q-d table are formed with $e_{0}^{(i)}=0, i=0, \ldots, n-2$, and $q_{1}^{(0)}=-b_{1}, q_{1}^{(i)}=$ $-b_{i+1} / b_{i}, i=1, \ldots, n-1$. New columns are formed as shown above, and the coefficients are, in sequence, the initial elements of the columns of the q-d table; that is, $a_{1}=q_{1}^{(0)}, a_{2}=e_{1}^{(0)}, a_{3}=q_{2}^{(0)}$ etc.

To use the above algorithm as a stable way of finding $\left\{a_{i}\right\}$ requires an accurate method for forming the leading diagonal of differences of the column $c_{i}=q_{1}^{(i)}$. Some methods which may be applicable are:

(i) If $c_{i}=1 /(i+a)$, then

$$
\Delta_{n-k-1}^{k}(c)=\frac{k !}{(n+a-1) \cdots(n+a+k-1)} .
$$

(ii) If $c_{i}=f(i)$, where $f$ is an analytic function whose circle of convergence about $i=n-1$ strictly includes the point $i=0$, then by Taylor series expansion of each value $f(n-1-j)$ about $(n-1)$,

$$
\Delta_{n-k-1}^{k}(c)=\sum_{i=k}^{\infty} \frac{f^{(i)}(n-1)}{i !} \sum_{j=0}^{k}\left(\begin{array}{l}
k \\
j
\end{array}\right)(-1)^{i} j^{i}
$$

All such series converge.

(iii) If $c_{i}=\int_{C} t^{i} f(t) d t$, then $\Delta_{n}^{k}(c)=\int_{C} t^{n-k}(t-1)^{k} f(t) d t$.

(iv) Any sequence $\left\{c_{i}\right\}$ which can be expressed as a number of sums, products, and quotients of sequences which can be dealt with by methods (i) to (iii) can have its differences formed by the processes described earlier. 
Example. Gargantini and Henrici [1] have extensively analyzed the stability problems in forming the continued fraction corresponding to the asymptotic expansion for large $z$ of the modified Bessel function $K_{0}(z)$. They considered three modifications of the q-d algorithm, but found only one feasible. This, which they called the incremental form, relied on the similarity between the q-d table for $K_{0}(z)$ and an explicitly known table. The arithmetic was performed on the differences.

\section{TABLE}

\section{Comparison of continued fraction coefficients for $K_{0}(z)$}

(1) given by Gargantini and Henrici [1]

(2) obtained using 8-figure precision and the difference-table algorithm

(3) obtained using 15-figure precision and the difference-table algorithm

$\begin{array}{cccc}n & (1) & (2) & (3) \\ 10 & 2.4175126 & 2.4175125 & 2.4175126 \\ 20 & 4.9120470 & 4.9120468 & 4.9120470 \\ 30 & 7.4094526 & 7.4094523 & 7.4094526 \\ 40 & 9.9075635 & 9.9078297 & 9.9078301 \\ 50 & - & 12.406680 & 12.406680 \\ 60 & - & 14.905804 & 14.905804 \\ 70 & - & 17.405103 & 17.405104\end{array}$

This method is equivalent to using extra precision; it reduces the errors which arise initially, but does not remove the tendency to amplify them. Consequently, as the authors found, calculations in double precision were satisfactory for only 20 coefficients, compared with 14 with no modification at all. Using triple precision, they calculated and tabulated 40 coefficients.

The asymptotic series used is that for

$$
e^{z} \sqrt{\frac{2}{\pi z}} K_{0}(z) \sim \sum_{n=0}^{\infty} \frac{(-1)^{n}}{2^{n} n !} \frac{\Gamma(n+1 / 2)^{2}}{\Gamma(1 / 2)} \frac{1}{z^{n}} .
$$

The ratios of successive coefficients are

$$
q_{1}^{(n)}=c_{n}=\frac{(2 n+1)^{2}}{8(n+1)}=n / 2+1 / 8(n+1), \quad n=0,1,2, \ldots
$$

Then

$$
\Delta_{n}^{1}\left(q_{1}\right)=1 / 2-1 / 8(n+1)(n+2), \quad n=0,1,2, \ldots,
$$

and for $k>1$,

$$
\Delta_{n}^{k}\left(q_{1}\right)=\frac{(-1)^{k} k ! n !}{8(n+k+1) !} .
$$

The algorithm described above was implemented to find the first 70 continued fraction coefficients. For comparison the work was done on a PDP-11 computer, 
working to about 8 significant figures, and on a Cyber-76, working to about 15 significant figures. The results agreed to seven significant figures. They were also consistent with the triple-precision results of Gargantini and Henrici up to the 39th value. A table of the results is shown.

\section{CSIRO}

Division of Mathematics and Statistics

P. O. Box 310,

South Melbourne, Victoria 3205, Australia

1. I. GARGANTINI \& P. HENRICI, "A continued fraction algorithm for the computation of higher transcendental functions in the complex plane," Math. Comp., v. 21, 1967, pp. 18-29.

2. P. HENRICI, "The quotient-difference algorithm," in Mathematical Methods for Digital Computers, Vol. 2 (A. Ralston \& H. S. Wilf, Eds.), Wiley, New York, 1967.

3. J. H. MCCABE \& J. A. MURPHY, "Continued fractions which correspond to power series expansions at two points," J. Inst. Math. Appl., v. 17, 1976, pp. 233-247.

4. L. M. MILNE-THOMPSON, The Calculus of Finite Differences, Macmillan, London, 1951. 